\title{
The Use of Ag-Au Alloy Catalysts to Control the Growth Mode for Si Nanowires
}

\author{
Yi-Chia Chou*, Mark C. Reuter**, Eric A. Stach* and Frances M. Ross** \\ *Purdue University, Department of Materials Science and Engineering, West Lafayette, IN 47907 \\ **IBM T. J. Watson Research Center, Yorktown Heights, NY 10598
}

For the growth of $\mathrm{Si}$ and $\mathrm{Ge}$ nanowires using metal catalysts, experimental and theoretical studies [1-4] have shown that the vapor-solid-solid (VSS) growth mode is more favorable than the vaporliquid-solid (VLS) growth mode for producing heterojunctions with sharp interfaces. This is because solid catalysts that have low solubility for the growth species do not act as a reservoir when switching between Si and Ge. However, achieving this benefit from VSS growth requires a catalyst that is convenient to use and that has a suitable eutectic temperature $\left(\mathrm{T}_{\mathrm{eu}}\right)$.

Here, we explore Ag-Au alloys for nanowire growth. Ag and $\mathrm{Au}$ are miscible and do not form intermetallic compounds; both have simple eutectic reactions with $\mathrm{Si}$, but the $\mathrm{T}_{\mathrm{eu}}$ of $\mathrm{Ag}$ and $\mathrm{Si}$ is higher than that of Au and $\mathrm{Si}$ [5]. Thus, we may expect Ag-Au alloys to form simple eutectics with $\mathrm{Si}$, and we may also expect to be able to tune $\mathrm{T}_{\text {eu }}$ by adjusting the Ag/Au ratio, allowing VSS growth at high enough temperatures to achieve reasonable growth rates. A similar approach has been described for Al-Au alloys [4], but Ag-Au is potentially more exciting for scale-up to standard chemical vapor deposition (CVD) reactors because Ag is more resistant than Al to oxidation.

In situ transmission electron microscopy movies of nanowires during growth [6] provide an ideal and direct way to test these ideas. For a particular Ag-Au alloy composition, we can obtain both VSS and VLS growth by varying the temperature. VLS Ag-Au Si nanowires resemble those reported for pure Au [6], but VSS nanowires (Fig. 1) exhibit hexagonally faceted catalysts and sidewalls with sawtooth faceting similar to that seen in VLS growth with Au [6]. The growth kinetics, determined directly from in situ movies (Fig. 2), also reflect the catalyst state. For an individual nanowire, we find substantial hysteresis in the solidification and melting temperatures of the catalyst, presumably due to undercooling. For a range of temperatures we can therefore achieve either VSS or VLS growth. Such experiments show that VSS growth is 3-4 times slower than VLS growth for a fixed composition of $\mathrm{AgAu}_{2}$. This may be expected because of lower adsorption probability on the solid surface [7]. Both VLS and VSS growth rates increase with temperature, and activation energies can be determined.

Based on in situ measurements, we will discuss the transition temperatures and the fascinating morphology changes for both catalysts and nanowires on switching between VLS and VSS growth. We will then compare growth morphology and kinetics for nanowires that were grown using the same metals but at higher pressures in an ex situ CVD growth chamber. We conclude by discussing the promise of $\mathrm{Ag}$-Au catalysts for $\mathrm{Si} / \mathrm{Ge}$ heterostructure formation. We believe that alloy catalysts in general open up a promising route towards control of nanowire and heterostructure growth and morphology.

\section{References}

[1] C.-Y. Wen, J. Tersoff, M. C. Reuter, E. A. Stach and F. M. Ross, Physical Review Letters 105 (2010) 195502. 
[2] N. Li, T. Y. Tan, U. Gösele, Applied Physics A 90 (2008) 591.

[3] C.-Y. Wen, M. C. Reuter, J. Tersoff, E. A. Stach and F. M. Ross, Nano Letters 10 (2010) 514.

[4] C.-Y. Wen, M. C. Reuter, J. Bruley, J. Tersoff, S. Kodambaka, E. A. Stach and F. M. Ross, Science 326 (2009) 1247.

[5] Materials Science International Team MSIT, and O. Fabrichnaya, A. Prince: $A g-A u-S i$ (Silver - Gold-Silicon). G. Effenberg, S. Ilyenko (ed.). SpringerMaterials - The Landolt-Börnstein Database.

[6] F. M. Ross, J. Tersoff, M. C. Reuter, Physical Review Letters 14 (2005) 146104.

[7] S. Kodambaka, J. Tersoff, M. C. Reuter and F. M. Ross, Science 316 (2007) 729.

[8] This work was partially funded by NSF under Award No. DMR-0907483. We acknowledge

C.-Y. Wen, K. B. Reuter and A. W. Ellis for their technical contributions to this project.
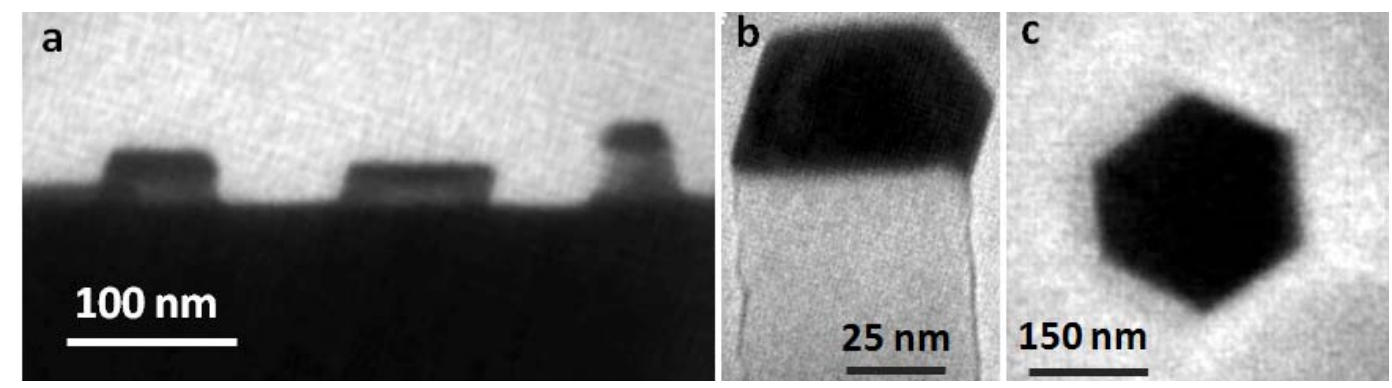

Fig. 1 In situ bright field TEM images of VSS Si nanowires grown with Ag-Au catalysts. Ag and $\mathrm{Au}$ were deposited in vacuum sequentially onto a Si substrate which was then heated to $400-510{ }^{\circ} \mathrm{C}$ and exposed to $1 \times 10^{-5}$ torr disilane. (a) View from the side showing three Si nanowires with $\mathrm{Ag}_{2} \mathrm{Au}$ catalysts at $510{ }^{\circ} \mathrm{C}$. (b) Higher magnification image of a single nanowire growing from a solid $\mathrm{Au}_{2} \mathrm{Ag}$ catalyst at $\sim 400{ }^{\circ} \mathrm{C}$. Sawtooth faceting is indicated on one sidewall (S). (c) Plan view image of a $\mathrm{Si}$ nanowire showing the hexagonal $\mathrm{Ag}_{2} \mathrm{Au}$ catalyst shape at $\sim 510{ }^{\circ} \mathrm{C}$.

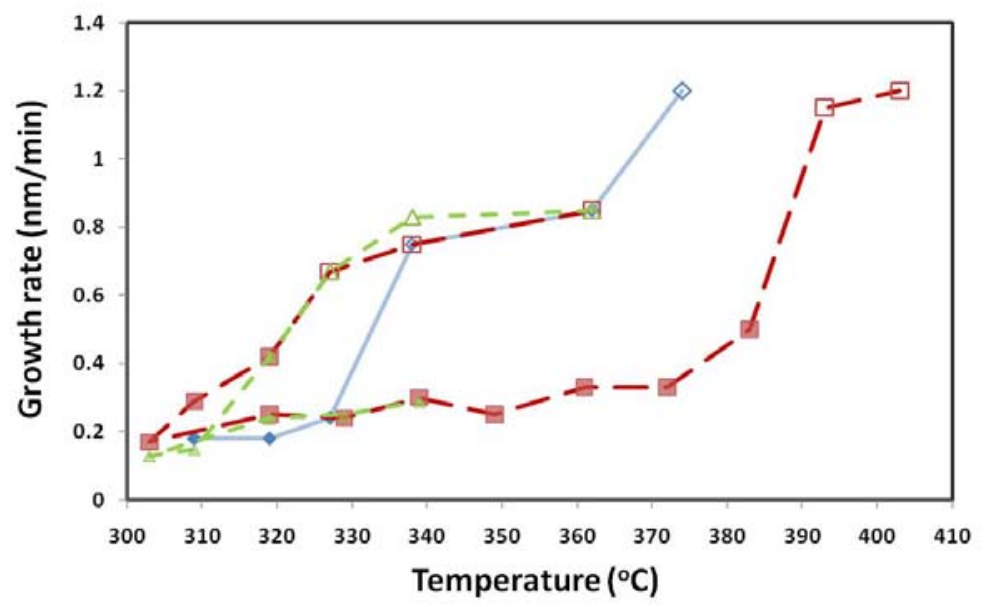

Fig. 2 The growth rates of three individual Si nanowires during growth from catalysts of nominal composition $\mathrm{AgAu}_{2}$ and exposed to $1 \times 10^{-5}$ torr disilane. In each case, the growth rate was measured over a range of temperatures while also noting whether the catalyst was solid or liquid. Open symbols represent liquid catalysts while solid ones represent solid catalysts. 